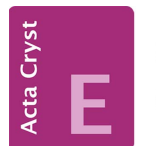
COMMUNICATIONS

ISSN 2056-9890

\section{Crystal structure of catena-poly $\left[\mathrm{bis}\left[\mu_{3}-\right.\right.$ 2-(2-nitrophenyl)acetato- $\left.\kappa^{3} O: O: O^{\prime}\right]$ - disilver(I)]}

Muhammad Danish, ${ }^{a}$ Muhammad Nawaz Tahir, ${ }^{\text {b* Sana }}$ Iftikhar $^{\mathrm{a}}$ and Nazir Ahmad ${ }^{\mathrm{C}}$

${ }^{a}$ Department of Chemistry, Institute of Natural Sciences, University of Gujrat, Gujrat 50700, Pakistan, ${ }^{\mathbf{b}}$ Department of Physics, University of Sargodha, Sargodha, Punjab, Pakistan, and ${ }^{\mathbf{c}}$ State Key Laboratory of Materials, Synthesis and New Technology, Wuhan University of Technology, Wuhan 430070, China. *Correspondence e-mail: dmntahir_uos@yahoo.com

Received 22 March 2015; accepted 17 April 2015

Edited by U. Flörke, University of Paderborn, Germany

The title compound, $\left[\mathrm{Ag}_{2}\left(\mathrm{C}_{8} \mathrm{H}_{6} \mathrm{NO}_{4}\right)_{2}\right]_{n}$, is a silver complex of 2-(2-nitrophenyl)acetic acid. The molecules are not conventional crystallographic inversion dimers but consist of two independent ligands and two $\mathrm{Ag}^{\mathrm{I}}$ ions, each with a distorted Tshaped coordination environment. The dihedral angles between acetate groups and the benzene rings are 51.1 (2) and $57.9(2)^{\circ}$. The nitro groups are oriented at dihedral angles of $23.6(5)$ and $32.3(3)^{\circ}$ with respect to the parent benzene rings. The dimers form polymeric chains along the $a$-axis direction. The Ag...Ag separation within a dimer is 2.8200 (5) and between symmetry-related dimers is 3.6182 (5) $\AA$. The polymeric chains are interlinked by $\mathrm{C}-\mathrm{H} \cdots \mathrm{O}$ hydrogen-bond interactions.

Keywords: crystal structure; silver(I) complex; 2-(2-nitrophenyl)acetic acid; hydrogen bonding.

CCDC reference: 1060272

\section{Related literature}

For related structures see: Danish et al. $(2011 a, b, 2015 a, b) ; \mathrm{Li}$ et al. (2011)

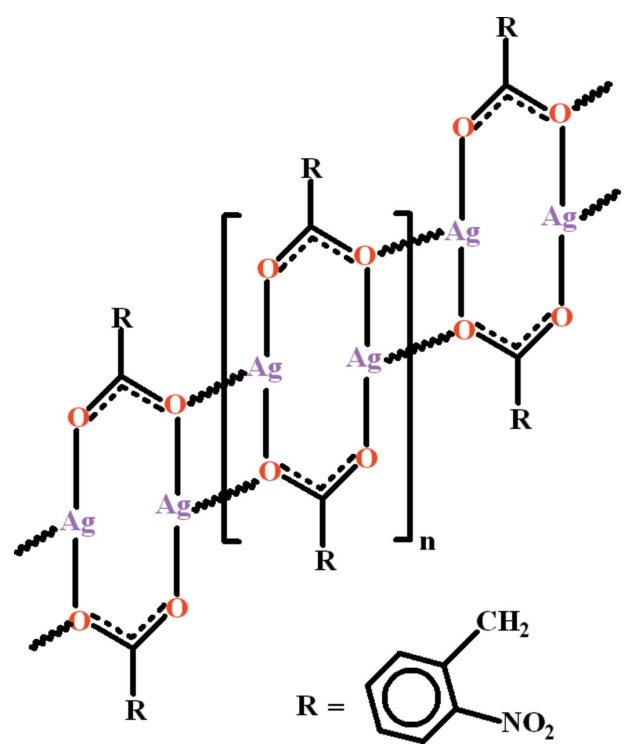

2. Experimental

2.1. Crystal data

$\left[\mathrm{Ag}_{2}\left(\mathrm{C}_{8} \mathrm{H}_{6} \mathrm{NO}_{4}\right)_{2}\right]$

$M_{r}=576.02$

Monoclinic, $P 2_{1} / c$

$a=5.5249$ (3) А

$b=15.8838(10) \AA$

$c=20.0257(11) \AA$

$\beta=96.853$ (3)

$$
\begin{aligned}
& V=1744.83(17) \AA^{3} \\
& Z=4 \\
& \text { Mo } K \alpha \text { radiation } \\
& \mu=2.30 \mathrm{~mm}^{-1} \\
& T=296 \mathrm{~K} \\
& 0.37 \times 0.22 \times 0.18 \mathrm{~mm}
\end{aligned}
$$

\subsection{Data collection}

Bruker Kappa APEXII CCD diffractometer

Absorption correction: multi-scan (SADABS; Bruker, 2005)

$T_{\text {min }}=0.485, T_{\text {max }}=0.682$

\subsection{Refinement}

$R\left[F^{2}>2 \sigma\left(F^{2}\right)\right]=0.033$

$w R\left(F^{2}\right)=0.066$

$S=1.10$

3781 reflections

253 parameters

$\mathrm{H}$-atom parameters constrained

$\Delta \rho_{\max }=0.47{\mathrm{e} \AA^{-3}}^{\circ}$

$\Delta \rho_{\min }=-0.55$ e $\AA^{-3}$

Table 1

Hydrogen-bond geometry $\left(\AA{ }^{\circ}\right)$.

\begin{tabular}{lllll}
\hline$D-\mathrm{H} \cdots A$ & $D-\mathrm{H}$ & $\mathrm{H} \cdots A$ & $D \cdots A$ & $D-\mathrm{H} \cdots A$ \\
\hline $\mathrm{C} 2-\mathrm{H} 2 B \cdots \mathrm{O} 4^{\mathrm{i}}$ & 0.97 & 2.53 & $3.312(6)$ & 138 \\
$\mathrm{C} 10-\mathrm{H} 10 B \cdots \mathrm{O} 8^{\mathrm{ii}}$ & 0.97 & 2.35 & $3.205(6)$ & 146 \\
$\mathrm{C} 6-\mathrm{H} 6 \cdots \mathrm{O}^{\text {ii }}$ & 0.93 & 2.48 & $3.322(6)$ & 151 \\
$\mathrm{C} 15-\mathrm{H} 15 \cdots \mathrm{O} 7^{\mathrm{iv}}$ & 0.93 & 2.59 & $3.268(6)$ & 130 \\
\hline Symmetry codes: (i) & $x-1, y, z ;$ & (ii) $x+1, y, z ;$ & (iii) $x,-y+\frac{1}{2}, z-\frac{1}{2} ; \quad$ (iv) \\
$-x+2, y-\frac{1}{2},-z+\frac{1}{2}$. & & & &
\end{tabular}

Data collection: APEX2 (Bruker, 2007); cell refinement: SAINT (Bruker, 2007); data reduction: $S A I N T$; program(s) used to solve structure: SHELXS97 (Sheldrick, 2008); program(s) used to refine structure: SHELXL2014 (Sheldrick, 2015); molecular graphics: 
ORTEP-3 for Windows (Farrugia, 2012) and PLATON (Spek, 2009); software used to prepare material for publication: Win $G X$ (Farrugia, 2012) and PLATON.

\section{Acknowledgements}

The authors acknowledge the provision of funds for the purchase of a diffractometer and encouragement by Dr Muhammad Akram Chaudhary, Vice Chancellor, University of Sargodha, Pakistan.

Supporting information for this paper is available from the IUCr electronic archives (Reference: FK2086).

\section{References}

Bruker (2005). SADABS. Bruker AXS Inc., Madison, Wisconsin, USA.

Bruker (2007). APEX2 and SAINT. Bruker AXS Inc., Madison, Wisconsin, USA.

Danish, M., Tahir, M. N., Ghafoor, S., Ahmad, N. \& Nisa, M. (2011a). Acta Cryst. E67, m734-m735.

Danish, M., Tahir, M. N., Ghafoor, S., Ahmad, N. \& Nisa, M. (2011b). Acta Cryst. E67, m938-m939.

Danish, M., Tahir, M. N., Iftikhar, S., Raza, M. A. \& Ashfaq, M. (2015a). Acta Cryst. E71, m52-m53.

Danish, M., Tahir, M. N., Iftikhar, S., Raza, M. A. \& Ashfaq, M. (2015b). Acta Cryst. E71, m59-m60.

Farrugia, L. J. (2012). J. Appl. Cryst. 45, 849-854.

Li, Y., Dong, X., Gou, Y., Jiang, Z. \& Zhu, H.-L. (2011). J. Coord. Chem. 64, $1663-1672$.

Sheldrick, G. M. (2008). Acta Cryst. A64, 112-122.

Sheldrick, G. M. (2015). Acta Cryst. C71, 3-8.

Spek, A. L. (2009). Acta Cryst. D65, 148-155. 


\section{supporting information}

Acta Cryst. (2015). E71, m118-m119 [https://doi.org/10.1107/S2056989015007616]

\section{Crystal structure of catena-poly [bis[ $\mu_{3}-2-(2-n i t r o p h e n y l) a c e t a t o-$ $\left.\kappa^{3} O: O: O^{\prime}\right]$ disilver(I)]}

\section{Muhammad Danish, Muhammad Nawaz Tahir, Sana Iftikhar and Nazir Ahmad}

\section{S1. Structural commentary}

We have reported the crystal structure of catena- poly[[trimethyltin(IV)]- $\mu$-2-(2-nitrophenyl)acetato- $\left.\kappa^{2} O: O^{\prime}\right]$ (Danish $e t$ al., 2015a) and tetra-aqua-bis-[2-(2-nitrophenyl)acetato- $\kappa O$ ]cobalt(II) (Danish et al., 2015b). The title silver complex (I), (Fig. 1) is in continuation of synthesizing various metal complexes with this legand and other studies of these complexes. We have also reported the crystal structures of catena-poly[bis-( $\mu 3-2$-methylbenzoato)disilver(I)] (Danish et al., 2011a) and catena-poly[bis-( $\mu 3$-2-methyl-3,5-dinitrobenzoato)disilver (I)] (Danish et al., 2011b) which are related to (I). Similarly, the crystal structures of bis( $N, N$-dimethylpyridin-4-amine)-((4-hydroxyphenyl) acetato)-silver dihydrate (Li et al., 2011), is related to the title compound.

In (I), the two ligands of (2-nitrophenyl)acetatic acid have been coordinated to to two silver ions making a dimer. The structural behaviour of both ligands is different. In one ligand the acetato moiety $A(\mathrm{O} 1 / \mathrm{C} 1 / \mathrm{C} 2 / \mathrm{O} 2)$ and benzene ring $B$ (C3-C8) are planar with r.m.s. deviation of 0.0041 and $0.0091 \AA$, respectively. The dihedral angle between $A / B$ is 57.87 $(17)^{\circ}$. The nitro group is oriented at a dihedtal angle of $23.6(5)^{\circ}$ with the parent benzene ring. In the second ligand the acetato moiety $C(\mathrm{O} 5 / \mathrm{C} 9 / \mathrm{C} 10 / \mathrm{O} 6)$ and benzene ring $D(\mathrm{C} 11-\mathrm{C} 16)$ are also planar with $\mathrm{r}$. $\mathrm{m}$. s. deviation of 0.0046 and $0.0147 \AA$, respectively. The dihedral angle between $C / D$ is $51.13(16)^{\circ}$. The adjacent nitro group makes dihedral angle of $32.3(3)^{\circ}$ with $D$. The central eight membered ring ( $\left.\mathrm{Ag} 1 / \mathrm{O} 1 / \mathrm{C} 1 / \mathrm{O} 2 / \mathrm{Ag} 2 / \mathrm{O} 5 / \mathrm{C} 9 / \mathrm{O} 6\right)$ is not exactly planar. The dimers are interlinked from opposite ends due to $\mathrm{Ag}-\mathrm{O}$ bonds in the form of one dimensional polymers extending along the $a$-axis. One of the $\mathrm{H}$-atoms of each methlenic group makes $\mathrm{H}$-bonding with adjacent nitro group of parental ligand in the onedimensional chain. The polymers are interlinked due to $\mathrm{C}-\mathrm{H} \cdots \mathrm{O}$ interactions, where $\mathrm{C}$-atoms are of benzene rings and $\mathrm{O}$-atoms are of nitro groups, therfore, stabilizing the molecules in the form of three dimensional polymeric network ultimately.

\section{S2. Synthesis and crystallization}

The sodium salt of (2-nitrophenyl)acetic acid was prepared in water with one molar ratio of (2-nitrophenyl)acetic acid and $\mathrm{Na}\left(\mathrm{HCO}_{3}\right)$. In this solution one mole of silver nitrate $\mathrm{AgNO}_{3}(1.08 \mathrm{~g})$ dissolved in water was added and stirred for 5 minutes. Curd white precipitate formed was dissolved by adding few drops of liquid ammonia and kept for crystallization in dark. Needle like colourless crystals were obtained after two weeks.

Yield: 45\% Melting Point: 395 K (Decomposes)

\section{S3. Refinement}

Crystal data, data collection and structure refinement details are summarized in Table 1. The H-atoms were positioned geometrically $(\mathrm{C}-\mathrm{H}=0.93-0.97 \AA)$ and refined riding on the carbon atoms with isotropic displacement parameters $\mathrm{U}_{\text {iso }}(\mathrm{H})=1.2 \mathrm{U}_{\mathrm{eq}}(\mathrm{C})$. 


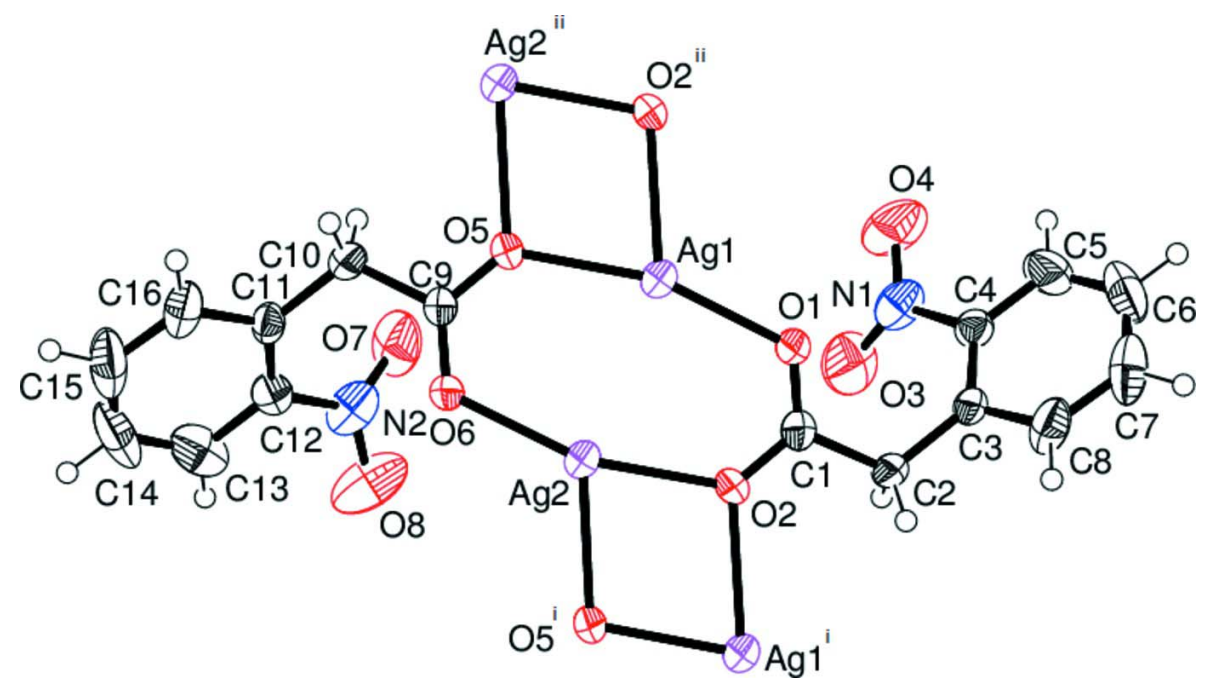

Figure 1

Molecular structure of the title compound. Anisotropic displacement ellipsoids are drawn at the $50 \%$ probability level. $\mathrm{H}$ atoms are shown by small circles of arbitrary radii.

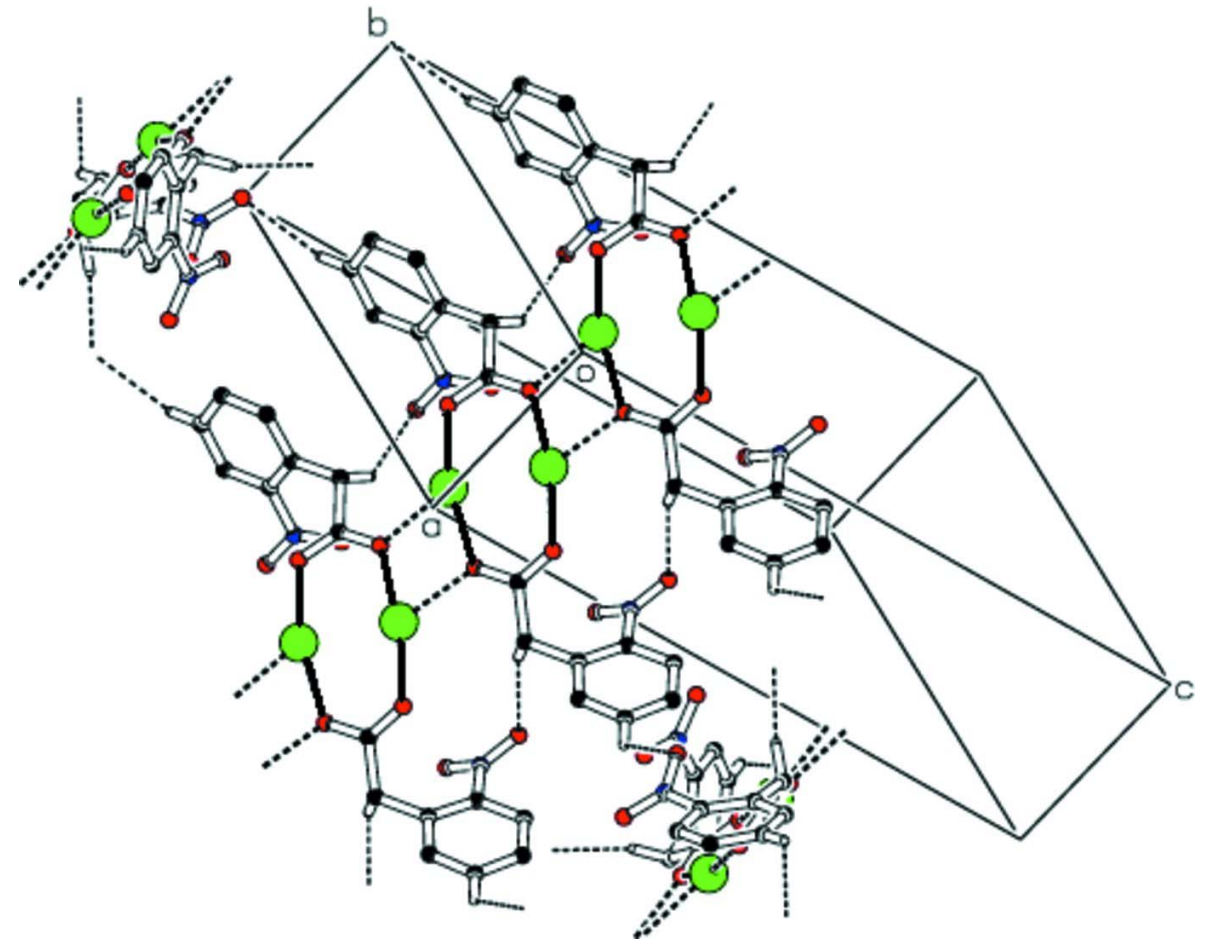

Figure 2

Crystal packing which shows that molecules form polymeric network due to interlinkage of dimers. The dimers are interlinked due to H-bondings. The $\mathrm{H}$-atoms not involved in H-bondings are omitted for clarity. 
catena-Poly[bis $\left[\mu_{3}-2-\left(2-\right.\right.$ nitrophenyl)acetato- $\left.\left.\kappa^{3} O: O: O^{\prime}\right] \operatorname{disilver}(\mathrm{I})\right]$

Crystal data

$$
\begin{aligned}
& {\left[\mathrm{Ag}_{2}\left(\mathrm{C}_{8} \mathrm{H}_{6} \mathrm{NO}_{4}\right)_{2}\right]} \\
& M_{r}=576.02 \\
& \text { Monoclinic, } P 2_{1} / c \\
& a=5.5249(3) \AA \\
& b=15.8838(10) \AA \\
& c=20.0257(11) \AA \\
& \beta=96.853(3)^{\circ} \\
& V=1744.83(17) \AA^{3} \\
& Z=4
\end{aligned}
$$

\section{Data collection}

\section{Bruker Kappa APEXII CCD} diffractometer

Radiation source: fine-focus sealed tube

Graphite monochromator

Detector resolution: 7.80 pixels $\mathrm{mm}^{-1}$

$\omega$ scans

Absorption correction: multi-scan

(SADABS; Bruker, 2005)

$T_{\min }=0.485, T_{\max }=0.682$

\section{Refinement}

Refinement on $F^{2}$

Least-squares matrix: full

$R\left[F^{2}>2 \sigma\left(F^{2}\right)\right]=0.033$

$w R\left(F^{2}\right)=0.066$

$S=1.10$

3781 reflections

253 parameters

0 restraints

Primary atom site location: structure-invariant direct methods

$$
F(000)=1120
$$

$D_{\mathrm{x}}=2.193 \mathrm{Mg} \mathrm{m}^{-3}$

Mo $K \alpha$ radiation, $\lambda=0.71073 \AA$

Cell parameters from 3113 reflections

$\theta=1.6-27.0^{\circ}$

$\mu=2.30 \mathrm{~mm}^{-1}$

$T=296 \mathrm{~K}$

Needle, colorless

$0.37 \times 0.22 \times 0.18 \mathrm{~mm}$

14170 measured reflections

3781 independent reflections

3113 reflections with $I>2 \sigma(I)$

$R_{\text {int }}=0.027$

$\theta_{\max }=27.0^{\circ}, \theta_{\min }=1.6^{\circ}$

$h=-7 \rightarrow 7$

$k=-20 \rightarrow 20$

$l=-25 \rightarrow 25$

Secondary atom site location: difference Fourier map

Hydrogen site location: inferred from

neighbouring sites

$\mathrm{H}$-atom parameters constrained

$w=1 /\left[\sigma^{2}\left(F_{\mathrm{o}}^{2}\right)+(0.0165 P)^{2}+3.0884 P\right]$

where $P=\left(F_{\mathrm{o}}^{2}+2 F_{\mathrm{c}}^{2}\right) / 3$

$(\Delta / \sigma)_{\max }<0.001$

$\Delta \rho_{\max }=0.47 \mathrm{e} \AA^{-3}$

$\Delta \rho_{\min }=-0.55$ e $\AA^{-3}$

Special details

Geometry. All e.s.d.'s (except the e.s.d. in the dihedral angle between two 1.s. planes) are estimated using the full covariance matrix. The cell e.s.d.'s are taken into account individually in the estimation of e.s.d.'s in distances, angles and torsion angles; correlations between e.s.d.'s in cell parameters are only used when they are defined by crystal symmetry. An approximate (isotropic) treatment of cell e.s.d.'s is used for estimating e.s.d.'s involving 1.s. planes.

Refinement. Refinement of $F^{2}$ against ALL reflections. The weighted $R$-factor $w R$ and goodness of fit $S$ are based on $F^{2}$, conventional $R$-factors $R$ are based on $F$, with $F$ set to zero for negative $F^{2}$. The threshold expression of $F^{2}>\sigma\left(F^{2}\right)$ is used only for calculating $R$-factors(gt) etc. and is not relevant to the choice of reflections for refinement. $R$-factors based on $F^{2}$ are statistically about twice as large as those based on $F$, and $R$-factors based on ALL data will be even larger.

Fractional atomic coordinates and isotropic or equivalent isotropic displacement parameters $\left(\AA^{2}\right)$

\begin{tabular}{lllll}
\hline & $x$ & $y$ & $z$ & $U_{\mathrm{iso}} * / U_{\text {eq }}$ \\
\hline Ag1 & $0.94971(5)$ & $0.08333(2)$ & $0.04106(2)$ & $0.04649(10)$ \\
Ag2 & $0.55095(5)$ & $-0.00701(2)$ & $0.08051(2)$ & $0.04710(10)$ \\
O1 & $0.6850(4)$ & $0.17796(16)$ & $0.00365(15)$ & $0.0446(7)$ \\
O2 & $0.3776(5)$ & $0.11268(17)$ & $0.04293(16)$ & $0.0507(8)$
\end{tabular}




$\begin{array}{lllll}\text { O3 } & 0.6222(7) & 0.3128(3) & 0.10446(17) & 0.0813(12) \\ \text { O4 } & 0.9451(7) & 0.3737(3) & 0.0785(2) & 0.0857(12) \\ \text { O5 } & 1.1302(5) & -0.02471(16) & 0.09716(15) & 0.0455(7) \\ \text { O6 } & 0.8165(4) & -0.09755(16) & 0.12586(14) & 0.0422(7) \\ \text { O7 } & 1.0110(7) & -0.0492(2) & 0.2652(2) & 0.0769(11) \\ \text { O8 } & 0.6646(7) & -0.0985(3) & 0.28321(19) & 0.0891(13) \\ \text { N1 } & 0.7356(7) & 0.3501(2) & 0.06550(19) & 0.0519(9) \\ \text { N2 } & 0.8701(7) & -0.1072(3) & 0.26878(18) & 0.0534(10) \\ \text { C1 } & 0.4719(7) & 0.1752(2) & 0.0172(2) & 0.0376(9) \\ \text { C2 } & 0.3050(7) & 0.2495(2) & 0.0012(2) & 0.0439(10) \\ \text { H2A } & 0.1657 & 0.2306 & -0.0292 & 0.053^{*} \\ \text { H2B } & 0.2445 & 0.2672 & 0.0426 & 0.053^{*} \\ \text { C3 } & 0.4109(6) & 0.3250(2) & -0.02943(19) & 0.0321(8) \\ \text { C4 } & 0.6114(7) & 0.3702(2) & -0.00200(19) & 0.0344(8) \\ \text { C5 } & 0.7012(8) & 0.4370(3) & -0.0343(3) & 0.0583(12) \\ \text { H5 } & 0.8407 & 0.4645 & -0.0149 & 0.070^{*} \\ \text { C6 } & 0.5883(11) & 0.4634(3) & -0.0944(3) & 0.0666(14) \\ \text { H6 } & 0.6486 & 0.5095 & -0.1158 & 0.080^{*} \\ \text { C7 } & 0.3877(11) & 0.4226(3) & -0.1231(2) & 0.0654(14) \\ \text { H7 } & 0.3080 & 0.4410 & -0.1640 & 0.079^{*} \\ \text { C8 } & 0.3015(8) & 0.3533(3) & -0.0914(2) & 0.0531(11) \\ \text { H8 } & 0.1660 & 0.3248 & -0.1122 & 0.064^{*} \\ \text { C9 } & 1.0388(6) & -0.0834(2) & 0.12825(19) & 0.0338(8) \\ \text { C10 } & 1.2235(7) & -0.1397(3) & 0.1690(2) & 0.0448(10) \\ \text { H10A } & 1.3176 & -0.1683 & 0.1379 & 0.054^{*} \\ \text { H10B } & 1.3352 & -0.1038 & 0.1972 & 0.054^{*} \\ \text { C11 } & 1.1271(7) & -0.2051(2) & 0.21313(18) & 0.0333(8) \\ \text { C12 } & 0.9564(7) & -0.1916(2) & 0.25696(18) & 0.0366(8) \\ \text { C13 } & 0.8654(9) & -0.2561(3) & 0.2933(2) & 0.0609(13) \\ \text { H13 } & 0.7455 & -0.2457 & 0.3211 & 0.073^{*} \\ \text { C14 } & 0.9568(12) & -0.3357(3) & 0.2873(3) & 0.0778(18) \\ \text { H14 } & 0.8973 & -0.3801 & 0.3108 & 0.093^{*} \\ \text { C15 } & 1.1325(11) & -0.3498(3) & 0.2474(3) & 0.0747(17) \\ \text { H15 } & 1.1997 & -0.4033 & 0.2453 & 0.090^{*} \\ \text { C16 } & 1.2119(8) & -0.2866(3) & 0.2102(2) & 0.0518(11) \\ \text { H16 } & 1.3281 & -0.2985 & 0.1815 & 0.062^{*} \\ & & & & \end{array}$

Atomic displacement parameters $\left(\AA^{2}\right)$

\begin{tabular}{lllllll}
\hline & $U^{11}$ & $U^{22}$ & $U^{33}$ & $U^{12}$ & $U^{13}$ & $U^{23}$ \\
\hline $\mathrm{Ag} 1$ & $0.02941(16)$ & $0.04213(18)$ & $0.0682(2)$ & $0.00658(13)$ & $0.00686(14)$ & $0.02080(15)$ \\
$\mathrm{Ag} 2$ & $0.02920(16)$ & $0.04436(18)$ & $0.0678(2)$ & $0.00628(13)$ & $0.00618(14)$ & $0.02365(16)$ \\
O1 & $0.0297(14)$ & $0.0374(15)$ & $0.069(2)$ & $0.0081(11)$ & $0.0160(13)$ & $0.0225(13)$ \\
O2 & $0.0301(14)$ & $0.0367(15)$ & $0.086(2)$ & $-0.0014(11)$ & $0.0084(14)$ & $0.0291(15)$ \\
O3 & $0.090(3)$ & $0.112(3)$ & $0.041(2)$ & $0.009(2)$ & $0.0057(19)$ & $0.018(2)$ \\
O4 & $0.054(2)$ & $0.101(3)$ & $0.093(3)$ & $-0.003(2)$ & $-0.031(2)$ & $-0.015(2)$ \\
O5 & $0.0312(14)$ & $0.0391(15)$ & $0.0670(19)$ & $0.0026(11)$ & $0.0095(13)$ & $0.0276(13)$ \\
O6 & $0.0265(14)$ & $0.0419(15)$ & $0.0569(18)$ & $-0.0024(11)$ & $-0.0010(12)$ & $0.0243(13)$
\end{tabular}




\begin{tabular}{lllllll} 
O7 & $0.093(3)$ & $0.0350(18)$ & $0.096(3)$ & $0.0016(18)$ & $-0.016(2)$ & $-0.0170(18)$ \\
O8 & $0.065(2)$ & $0.135(4)$ & $0.065(2)$ & $0.032(2)$ & $-0.0004(19)$ & $-0.044(2)$ \\
N1 & $0.050(2)$ & $0.053(2)$ & $0.049(2)$ & $0.0084(18)$ & $-0.0069(19)$ & $-0.0121(18)$ \\
N2 & $0.056(2)$ & $0.059(2)$ & $0.042(2)$ & $0.0120(19)$ & $-0.0094(18)$ & $-0.0205(18)$ \\
C1 & $0.035(2)$ & $0.0286(19)$ & $0.049(2)$ & $0.0023(15)$ & $0.0027(17)$ & $0.0075(16)$ \\
C2 & $0.0245(19)$ & $0.032(2)$ & $0.075(3)$ & $0.0038(15)$ & $0.0060(19)$ & $0.0145(19)$ \\
C3 & $0.0283(18)$ & $0.0251(17)$ & $0.043(2)$ & $0.0057(14)$ & $0.0039(16)$ & $0.0032(15)$ \\
C4 & $0.0321(19)$ & $0.0316(19)$ & $0.039(2)$ & $0.0009(15)$ & $0.0023(16)$ & $-0.0012(15)$ \\
C5 & $0.054(3)$ & $0.042(2)$ & $0.081(4)$ & $-0.013(2)$ & $0.012(3)$ & $0.004(2)$ \\
C6 & $0.096(4)$ & $0.039(3)$ & $0.070(4)$ & $0.002(3)$ & $0.033(3)$ & $0.018(2)$ \\
C7 & $0.102(4)$ & $0.053(3)$ & $0.040(3)$ & $0.024(3)$ & $0.002(3)$ & $0.013(2)$ \\
C8 & $0.058(3)$ & $0.041(2)$ & $0.053(3)$ & $0.008(2)$ & $-0.019(2)$ & $0.001(2)$ \\
C9 & $0.0315(19)$ & $0.0311(19)$ & $0.038(2)$ & $0.0021(15)$ & $0.0025(16)$ & $0.0100(16)$ \\
C10 & $0.029(2)$ & $0.051(2)$ & $0.055(3)$ & $0.0056(17)$ & $0.0051(18)$ & $0.022(2)$ \\
C11 & $0.035(2)$ & $0.0276(18)$ & $0.035(2)$ & $0.0015(14)$ & $-0.0060(16)$ & $0.0048(15)$ \\
C12 & $0.040(2)$ & $0.038(2)$ & $0.030(2)$ & $-0.0071(16)$ & $-0.0068(16)$ & $-0.0018(15)$ \\
C13 & $0.071(3)$ & $0.081(4)$ & $0.029(2)$ & $-0.024(3)$ & $0.000(2)$ & $0.005(2)$ \\
C14 & $0.111(5)$ & $0.052(3)$ & $0.063(4)$ & $-0.038(3)$ & $-0.021(3)$ & $0.027(3)$ \\
C15 & $0.103(5)$ & $0.029(2)$ & $0.083(4)$ & $-0.002(3)$ & $-0.027(3)$ & $0.011(2)$ \\
C16 & $0.061(3)$ & $0.037(2)$ & $0.054(3)$ & $0.013(2)$ & $-0.009(2)$ & $0.002(2)$ \\
& & & & & & \\
\hline
\end{tabular}

Geometric parameters $\left(\AA,{ }^{o}\right)$

\begin{tabular}{llll}
\hline $\mathrm{Ag} 1-\mathrm{O} 1$ & $2.167(2)$ & $\mathrm{C} 3-\mathrm{C} 4$ & $1.379(5)$ \\
$\mathrm{Ag} 1-\mathrm{O} 5$ & $2.221(2)$ & $\mathrm{C} 3-\mathrm{C} 8$ & $1.388(5)$ \\
$\mathrm{Ag} 1-\mathrm{O} 2^{\mathrm{i}}$ & $2.405(3)$ & $\mathrm{C} 4-\mathrm{C} 5$ & $1.365(5)$ \\
$\mathrm{Ag} 1-\mathrm{Ag} 2$ & $2.8200(4)$ & $\mathrm{C} 5-\mathrm{C} 6$ & $1.354(7)$ \\
$\mathrm{Ag} 1-\mathrm{Ag} 1^{\mathrm{ii}}$ & $3.1998(7)$ & $\mathrm{C} 5-\mathrm{H} 5$ & 0.9300 \\
$\mathrm{Ag} 2-\mathrm{O} 6$ & $2.174(2)$ & $\mathrm{C} 6-\mathrm{C} 7$ & $1.351(7)$ \\
$\mathrm{Ag} 2-\mathrm{O} 2$ & $2.219(3)$ & $\mathrm{C} 6-\mathrm{H} 6$ & 0.9300 \\
$\mathrm{Ag} 2-\mathrm{O} 5^{\mathrm{iii}}$ & $2.404(2)$ & $\mathrm{C} 7-\mathrm{C} 8$ & $1.384(7)$ \\
$\mathrm{Ag} 2-\mathrm{Ag} 2^{\mathrm{iv}}$ & $3.2140(7)$ & $\mathrm{C} 7-\mathrm{H} 7$ & 0.9300 \\
$\mathrm{O} 1-\mathrm{C} 1$ & $1.241(4)$ & $\mathrm{C} 8-\mathrm{H} 8$ & 0.9300 \\
$\mathrm{O} 2-\mathrm{C} 1$ & $1.259(4)$ & $\mathrm{C} 9-\mathrm{C} 10$ & $1.518(5)$ \\
$\mathrm{O} 2-\mathrm{Ag} 1^{\mathrm{iii}}$ & $2.405(3)$ & $\mathrm{C} 10-\mathrm{C} 11$ & $1.503(5)$ \\
$\mathrm{O} 3-\mathrm{N} 1$ & $1.211(5)$ & $\mathrm{C} 10-\mathrm{H} 10 \mathrm{~A}$ & 0.9700 \\
$\mathrm{O} 4-\mathrm{N} 1$ & $1.215(5)$ & $\mathrm{C} 10-\mathrm{H} 10 \mathrm{~B}$ & 0.9700 \\
$\mathrm{O} 5-\mathrm{C} 9$ & $1.260(4)$ & $\mathrm{C} 11-\mathrm{C} 12$ & $1.380(5)$ \\
$\mathrm{O} 5-\mathrm{Ag} 2^{\mathrm{i}}$ & $2.404(2)$ & $\mathrm{C} 11-\mathrm{C} 16$ & $1.380(5)$ \\
$\mathrm{O} 6-\mathrm{C} 9$ & $1.244(4)$ & $\mathrm{C} 12-\mathrm{C} 13$ & $1.386(6)$ \\
$\mathrm{O} 7-\mathrm{N} 2$ & $1.214(5)$ & $\mathrm{C} 13-\mathrm{C} 14$ & $1.373(8)$ \\
$\mathrm{O} 8-\mathrm{N} 2$ & $1.212(5)$ & $\mathrm{C} 13-\mathrm{H} 13$ & 0.9300 \\
$\mathrm{~N} 1-\mathrm{C} 4$ & $1.476(5)$ & $\mathrm{C} 14-\mathrm{C} 15$ & $1.348(8)$ \\
$\mathrm{N} 2-\mathrm{C} 12$ & $1.451(5)$ & $\mathrm{C} 14-\mathrm{H} 14$ & 0.9300 \\
$\mathrm{C} 1-\mathrm{C} 2$ & $1.509(5)$ & $\mathrm{C} 15-\mathrm{C} 16$ & $1.354(7)$ \\
$\mathrm{C} 2-\mathrm{C} 3$ & $1.498(5)$ & $\mathrm{C} 15-\mathrm{H} 15$ & 0.9300 \\
$\mathrm{C} 2-\mathrm{H} 2 \mathrm{~A}$ & 0.9700 & $\mathrm{C} 16-\mathrm{H} 16$ & 0.9300 \\
$\mathrm{C} 2-\mathrm{H} 2 \mathrm{~B}$ & 0.9700 & &
\end{tabular}




\begin{tabular}{|c|c|c|c|}
\hline $\mathrm{O} 1-\mathrm{Ag} 1-\mathrm{O} 5$ & $162.60(9)$ & $\mathrm{C} 4-\mathrm{C} 3-\mathrm{C} 2$ & $126.1(3)$ \\
\hline $\mathrm{O} 1-\mathrm{Ag} 1-\mathrm{O} 2^{\mathrm{i}}$ & $119.49(9)$ & $\mathrm{C} 8-\mathrm{C} 3-\mathrm{C} 2$ & $118.4(4)$ \\
\hline $\mathrm{O} 5-\mathrm{Ag} 1-\mathrm{O} 2^{\mathrm{i}}$ & $76.16(9)$ & $\mathrm{C} 5-\mathrm{C} 4-\mathrm{C} 3$ & $122.4(4)$ \\
\hline $\mathrm{O} 1-\mathrm{Ag} 1-\mathrm{Ag} 2$ & $86.12(7)$ & $\mathrm{C} 5-\mathrm{C} 4-\mathrm{N} 1$ & $116.5(4)$ \\
\hline $\mathrm{O} 5-\mathrm{Ag} 1-\mathrm{Ag} 2$ & $77.51(6)$ & $\mathrm{C} 3-\mathrm{C} 4-\mathrm{N} 1$ & $121.0(3)$ \\
\hline $\mathrm{O} 2 \mathrm{i}-\mathrm{Ag} 1-\mathrm{Ag} 2$ & $153.31(6)$ & $\mathrm{C} 6-\mathrm{C} 5-\mathrm{C} 4$ & $120.4(4)$ \\
\hline $\mathrm{O} 1-\mathrm{Ag} 1-\mathrm{Ag} 1^{\mathrm{ii}}$ & $123.20(8)$ & $\mathrm{C} 6-\mathrm{C} 5-\mathrm{H} 5$ & 119.8 \\
\hline $\mathrm{O} 5-\mathrm{Ag} 1-\mathrm{Ag} 1^{\mathrm{ii}}$ & $61.69(8)$ & $\mathrm{C} 4-\mathrm{C} 5-\mathrm{H} 5$ & 119.8 \\
\hline $\mathrm{O} 2^{\mathrm{i}}-\mathrm{Ag} 1-\mathrm{Ag} 1^{\mathrm{ii}}$ & $86.43(8)$ & $\mathrm{C} 7-\mathrm{C} 6-\mathrm{C} 5$ & $119.8(4)$ \\
\hline $\mathrm{Ag} 2-\mathrm{Ag} 1-\mathrm{Ag} 1^{\mathrm{ii}}$ & $84.972(15)$ & $\mathrm{C} 7-\mathrm{C} 6-\mathrm{H} 6$ & 120.1 \\
\hline $\mathrm{O} 6-\mathrm{Ag} 2-\mathrm{O} 2$ & $161.91(10)$ & $\mathrm{C} 5-\mathrm{C} 6-\mathrm{H} 6$ & 120.1 \\
\hline $\mathrm{O} 6-\mathrm{Ag} 2-\mathrm{O} 5^{\mathrm{iii}}$ & $118.69(9)$ & $\mathrm{C} 6-\mathrm{C} 7-\mathrm{C} 8$ & $119.7(4)$ \\
\hline $\mathrm{O} 2-\mathrm{Ag} 2-\mathrm{O} 5^{\mathrm{iii}}$ & $76.23(9)$ & $\mathrm{C} 6-\mathrm{C} 7-\mathrm{H} 7$ & 120.2 \\
\hline $\mathrm{O} 6-\mathrm{Ag} 2-\mathrm{Ag} 1$ & $86.72(6)$ & $\mathrm{C} 8-\mathrm{C} 7-\mathrm{H} 7$ & 120.2 \\
\hline $\mathrm{O} 2-\mathrm{Ag} 2-\mathrm{Ag} 1$ & $77.83(6)$ & $\mathrm{C} 7-\mathrm{C} 8-\mathrm{C} 3$ & $122.1(4)$ \\
\hline $\mathrm{O} 5^{\mathrm{iii}}-\mathrm{Ag} 2-\mathrm{Ag} 1$ & $154.04(6)$ & $\mathrm{C} 7-\mathrm{C} 8-\mathrm{H} 8$ & 119.0 \\
\hline $\mathrm{O} 6-\mathrm{Ag} 2-\mathrm{Ag} 2^{\mathrm{iv}}$ & $119.58(8)$ & $\mathrm{C} 3-\mathrm{C} 8-\mathrm{H} 8$ & 119.0 \\
\hline $\mathrm{O} 2-\mathrm{Ag} 2-\mathrm{Ag} 2^{\mathrm{iv}}$ & $65.40(9)$ & $\mathrm{O} 6-\mathrm{C} 9-\mathrm{O} 5$ & $124.5(3)$ \\
\hline $\mathrm{O} 5^{\mathrm{iii}}-\mathrm{Ag} 2-\mathrm{Ag} 2^{\mathrm{iv}}$ & $95.20(7)$ & $\mathrm{O} 6-\mathrm{C} 9-\mathrm{C} 10$ & $120.8(3)$ \\
\hline $\mathrm{Ag} 1-\mathrm{Ag} 2-\mathrm{Ag} 2^{\mathrm{iv}}$ & $74.507(13)$ & $\mathrm{O} 5-\mathrm{C} 9-\mathrm{C} 10$ & $114.7(3)$ \\
\hline $\mathrm{C} 1-\mathrm{O} 1-\mathrm{Ag} 1$ & $121.2(2)$ & $\mathrm{C} 11-\mathrm{C} 10-\mathrm{C} 9$ & $117.4(3)$ \\
\hline $\mathrm{C} 1-\mathrm{O} 2-\mathrm{Ag} 2$ & $129.1(2)$ & $\mathrm{C} 11-\mathrm{C} 10-\mathrm{H} 10 \mathrm{~A}$ & 107.9 \\
\hline $\mathrm{C} 1-\mathrm{O} 2-\mathrm{Ag} 1^{\mathrm{iii}}$ & $126.9(2)$ & $\mathrm{C} 9-\mathrm{C} 10-\mathrm{H} 10 \mathrm{~A}$ & 107.9 \\
\hline $\mathrm{Ag} 2-\mathrm{O} 2-\mathrm{Ag} 1^{\mathrm{iii}}$ & $102.88(10)$ & $\mathrm{C} 11-\mathrm{C} 10-\mathrm{H} 10 \mathrm{~B}$ & 107.9 \\
\hline $\mathrm{C} 9-\mathrm{O} 5-\mathrm{Ag} 1$ & $129.8(2)$ & $\mathrm{C} 9-\mathrm{C} 10-\mathrm{H} 10 \mathrm{~B}$ & 108.0 \\
\hline $\mathrm{C} 9-\mathrm{O} 5-\mathrm{Ag} 2^{\mathrm{i}}$ & $127.3(2)$ & $\mathrm{H} 10 \mathrm{~A}-\mathrm{C} 10-\mathrm{H} 10 \mathrm{~B}$ & 107.2 \\
\hline $\mathrm{Ag} 1-\mathrm{O} 5-\mathrm{Ag} 2^{\mathrm{i}}$ & $102.86(9)$ & $\mathrm{C} 12-\mathrm{C} 11-\mathrm{C} 16$ & $115.8(4)$ \\
\hline C9-O6-Ag2 & $120.7(2)$ & $\mathrm{C} 12-\mathrm{C} 11-\mathrm{C} 10$ & $125.7(3)$ \\
\hline $\mathrm{O} 3-\mathrm{N} 1-\mathrm{O} 4$ & $124.4(4)$ & $\mathrm{C} 16-\mathrm{C} 11-\mathrm{C} 10$ & $118.5(4)$ \\
\hline $\mathrm{O} 3-\mathrm{N} 1-\mathrm{C} 4$ & $118.4(4)$ & $\mathrm{C} 11-\mathrm{C} 12-\mathrm{C} 13$ & $122.5(4)$ \\
\hline $\mathrm{O} 4-\mathrm{N} 1-\mathrm{C} 4$ & $117.1(4)$ & $\mathrm{C} 11-\mathrm{C} 12-\mathrm{N} 2$ & $120.7(4)$ \\
\hline $\mathrm{O} 8-\mathrm{N} 2-\mathrm{O} 7$ & $123.6(4)$ & $\mathrm{C} 13-\mathrm{C} 12-\mathrm{N} 2$ & $116.7(4)$ \\
\hline $\mathrm{O} 8-\mathrm{N} 2-\mathrm{C} 12$ & $118.7(4)$ & $\mathrm{C} 14-\mathrm{C} 13-\mathrm{C} 12$ & $118.3(5)$ \\
\hline $\mathrm{O} 7-\mathrm{N} 2-\mathrm{C} 12$ & $117.7(4)$ & $\mathrm{C} 14-\mathrm{C} 13-\mathrm{H} 13$ & 120.8 \\
\hline $\mathrm{O} 1-\mathrm{C} 1-\mathrm{O} 2$ & $124.6(3)$ & $\mathrm{C} 12-\mathrm{C} 13-\mathrm{H} 13$ & 120.8 \\
\hline $\mathrm{O} 1-\mathrm{C} 1-\mathrm{C} 2$ & $119.8(3)$ & $\mathrm{C} 15-\mathrm{C} 14-\mathrm{C} 13$ & $120.2(5)$ \\
\hline $\mathrm{O} 2-\mathrm{C} 1-\mathrm{C} 2$ & $115.6(3)$ & $\mathrm{C} 15-\mathrm{C} 14-\mathrm{H} 14$ & 119.9 \\
\hline $\mathrm{C} 3-\mathrm{C} 2-\mathrm{C} 1$ & $117.0(3)$ & $\mathrm{C} 13-\mathrm{C} 14-\mathrm{H} 14$ & 119.9 \\
\hline $\mathrm{C} 3-\mathrm{C} 2-\mathrm{H} 2 \mathrm{~A}$ & 108.0 & $\mathrm{C} 14-\mathrm{C} 15-\mathrm{C} 16$ & $120.4(5)$ \\
\hline $\mathrm{C} 1-\mathrm{C} 2-\mathrm{H} 2 \mathrm{~A}$ & 108.0 & $\mathrm{C} 14-\mathrm{C} 15-\mathrm{H} 15$ & 119.8 \\
\hline $\mathrm{C} 3-\mathrm{C} 2-\mathrm{H} 2 \mathrm{~B}$ & 108.0 & $\mathrm{C} 16-\mathrm{C} 15-\mathrm{H} 15$ & 119.8 \\
\hline $\mathrm{C} 1-\mathrm{C} 2-\mathrm{H} 2 \mathrm{~B}$ & 108.0 & $\mathrm{C} 15-\mathrm{C} 16-\mathrm{C} 11$ & $122.5(5)$ \\
\hline $\mathrm{H} 2 \mathrm{~A}-\mathrm{C} 2-\mathrm{H} 2 \mathrm{~B}$ & 107.3 & $\mathrm{C} 15-\mathrm{C} 16-\mathrm{H} 16$ & 118.7 \\
\hline $\mathrm{C} 4-\mathrm{C} 3-\mathrm{C} 8$ & $115.5(3)$ & $\mathrm{C} 11-\mathrm{C} 16-\mathrm{H} 16$ & 118.7 \\
\hline $\mathrm{Ag} 1-\mathrm{O} 1-\mathrm{C} 1-\mathrm{O} 2$ & $-12.5(6)$ & $\mathrm{Ag} 2-\mathrm{O} 6-\mathrm{C} 9-\mathrm{O} 5$ & $-11.1(5)$ \\
\hline $\mathrm{Ag} 1-\mathrm{O} 1-\mathrm{C} 1-\mathrm{C} 2$ & $169.0(3)$ & $\mathrm{Ag} 2-\mathrm{O} 6-\mathrm{C} 9-\mathrm{C} 10$ & $170.6(3)$ \\
\hline
\end{tabular}




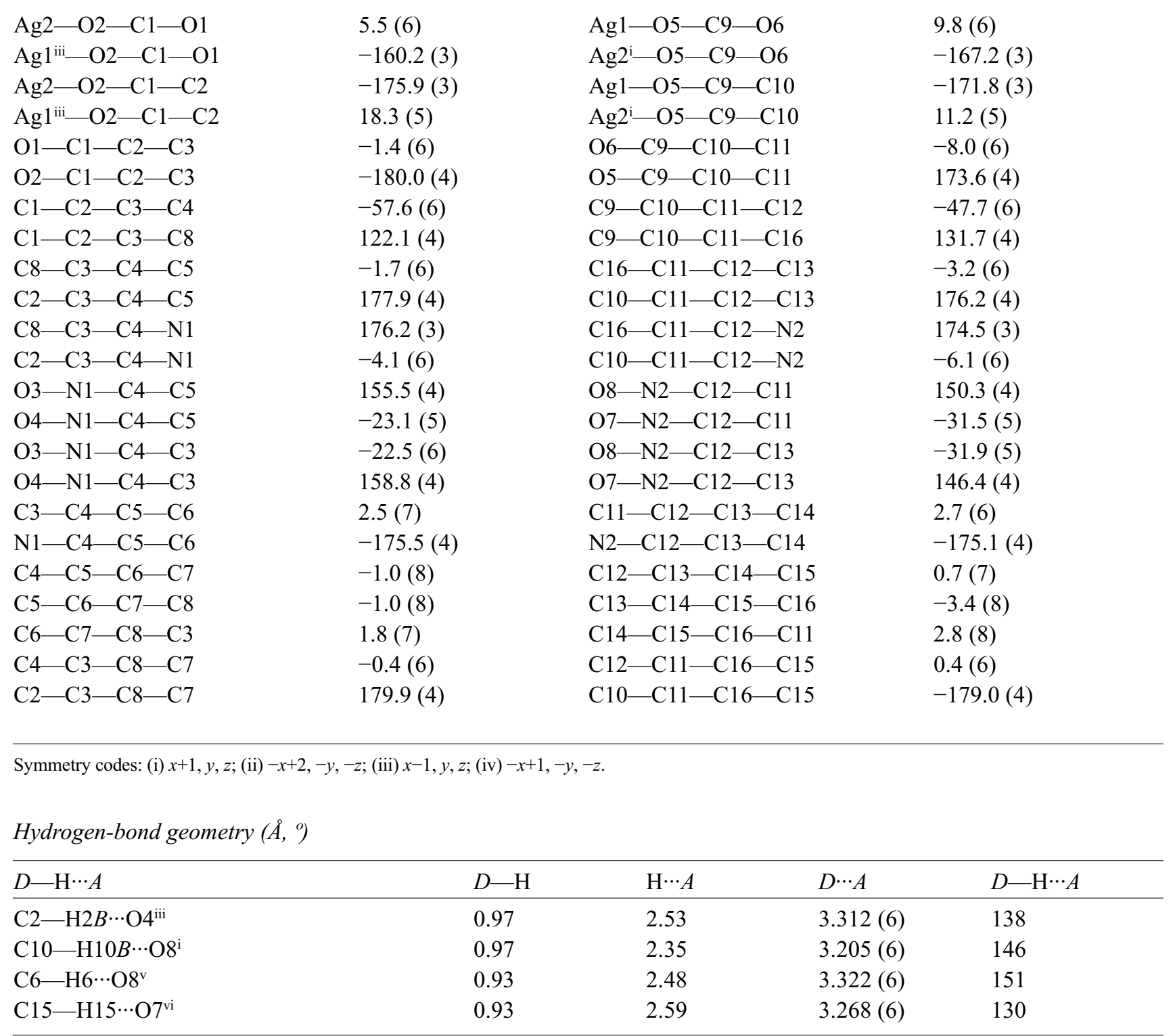

Symmetry codes: (i) $x+1, y, z$; (iii) $x-1, y, z$; (v) $x,-y+1 / 2, z-1 / 2$; (vi) $-x+2, y-1 / 2,-z+1 / 2$. 